Revue

de I'histoire des religions

\section{Revue de l'histoire des religions}

$4 \mid 2013$

Sermo mysticus Mystique et langage entre Moyen Âge et époque moderne

\title{
La Compilatio mystica ou le doux miel de la mystique rhénane
}

The Compilatio mystica or the sweet honey of Rhineland mysticism

Maxime Mauriège

\section{OpenEdition}

\section{Journals}

Édition électronique

URL : http://journals.openedition.org/rhr/8173

DOI : $10.4000 /$ rhr.8173

ISSN : 2105-2573

Éditeur

Armand Colin

Édition imprimée

Date de publication : 1 décembre 2013

Pagination : 485-507

ISBN : $978-2200928667$

ISSN : 0035-1423

Référence électronique

Maxime Mauriège, "La Compilatio mystica ou le doux miel de la mystique rhénane », Revue de l'histoire des religions [En ligne], 4 | 2013, mis en ligne le 01 décembre 2016, consulté le 02 mai 2019. URL http://journals.openedition.org/rhr/8173 ; DOI : 10.4000/rhr.8173 
MAXIME MAURIÈGE

Thomas-Institut, Cologne

\section{La Compilatio mystica ou le doux miel de la mystique rhénane}

L'étude est centrée sur la réception de l'enseignement de la mystique allemande médiévale dans la Compilatio mystica, traité-mosaïque de la fin du XIV siècle, en étayant les raisons de son rattachement à la mystique dite "rhénane », représentée par Maître Eckhart et ses disciples. L'étude examine d'abord la manière dont cette compilation fut jadis considérée, grâce à quelques notices de manuscrits permettant de mieux cerner le phénomène de sa réception. Vient ensuite un aperçu schématique du contenu riche et varié de cette "mosä̈que", en prêtant attention à sa cohérence "sys-thématique», au langage et au style caractéristiques, puis aux auteurs mystiques compilés, sans oublier le poids des auctoritates et de l'érudition scolastique dans ce Lehrsystem en moyen haut-allemand.

\section{The Compilatio mystica or the sweet honey of Rhineland mysticism}

The article studies the reception of medieval German mysticism in the Compilatio mystica, a mosaic treatise from the end of the 14th century, in backing up the reasons of its attachment to so-called "Rhineland" mysticism, represented by Meister Eckhart and his followers. The study begins by considering how this compilation was regarded formerly through a few marginal notes in some manuscripts, which help to better understand its reception. This is followed by a schematic overview of the rich and diverse contents of this "mosaic", focusing on its sys-thematic coherence and on its characteristic language and style, and then on the mystical authors included in the compilation. The weight of the auctoritates and of scholastic erudition in this Lehrsystem in Middle High German is also taken into account. 
Au début de la seconde partie de la Vita du bienheureux Henri Suso, Elsbeth Stagel - «une fille spirituelle appartenant à l'Ordre des Prêcheurs» († vers 1360) - nous est présentée comme une religieuse si avide d'instruction sur la doctrine des maîtres de la vie spirituelle, qu'elle copiait pieusement tout enseignement profitable à l'édification d'elle-même et de son prochain, «comme les abeilles industrieuses qui puisent à des fleurs variées pour récolter le doux miel ${ }^{1}$. Cette métaphore répandue traduit ici l'intérêt et l'activité littéraires de la célèbre nonne du couvent de Töss² ${ }^{2}$ mais également, à travers elle, de toutes ces moniales dominicaines de la grande vallée du $\mathrm{Rhin}^{3}$, qui employèrent notamment leur zèle à copier (et, le cas échéant, à traduire) des passages choisis parmi diverses sources de la spiritualité chrétienne médiévale - en commençant, comme

1. Seuses Leben, c. 33, éd. Karl Bihlmeyer, dans Heinrich Seuse, Deutsche Schriften, Stuttgart, Kohhammer, 1907 [réimpression anastatique Francfort, Minerva, 1961], p. 96, 5-16; trad. J. Ancelet-Hustache, dans Henri Suso, Euvres complètes, Paris, Seuil, 1977, p. 227. L'ensemble du passage (éd. K. Bihlmeyer, p. 96, 5-16) se retrouve dans les Vies des sœurs de Töss, un recueil de biographies rédigé vers 1340, dont on attribue la paternité littéraire à Elsbeth Stagel. On constate toutefois que la présentation de celle-ci dans la Vita de Suso est allégée d'une remarque essentielle et s'en trouve appauvrie. En effet, ladite chronique stipule en outre que «l'abeille» de Töss «profita de la connaissance de plusieurs sages érudits et saints amis de Dieu, spécialement des bienheureux frères qui appartenaient comme elle au saint ordre des Prêcheurs; parmi ceux-ci, elle profita de la connaissance du saint et éminent maître, frère Eckhart, duquel elle reçut ainsi que de plusieurs autres - tant de bons et utiles enseignements » (Das Leben der Schwestern zu Töß beschrieben von Elsbet Stagel samt der Vorrede von Johannes Meier und dem Leben der Prinzessin Elisabet von Ungarn, éd. Ferdinand Vetter, Berlin, Weidmann, 1906 [Deutsche Texte des Mittelalters, 6], p. 4, 6-10). L'ajout est ici capital, puisqu'il témoigne explicitement et contextuellement de l'influence prééminente qu'exerça alors l'enseignement de Maître Eckhart sur l'orientation spirituelle des moniales dominicaines allemandes.

2. Voir Burkhard Hasebrink, «Zersetzung? Eine Neubewertung der Eckhartkompilation in Spamers Mosaiktraktaten», dans Kaspar Elm (éd.), Literarische Formen des Mittelalters: Florilegien, Kompilationen, Kollektionen, Wiesbaden, Harrassowitz, 2000 (Wolfenbütteler Mittelalter-Studien, 15), p. 73-90, ici p. 79 et n. 27; Hans-Jochen Schiewer, «Möglichkeiten und Grenzen schreibender Ordensfrauen im Spätmittelalter», dans Barbara Helbing, Magdalen Bless-Grabher, Ines Buhofer (éd.), Bettelorden, Bruderschaften und Beginen in Zürich: Stadtkultur und Seelenheil im Mittelalter, Zürich, Neue Züricher Zeitung, 2002 (Beiträge zur Hagiographie, 3), p. 179-187, ici p. 185-186.

3. Voir, entre autres, Rémy Valléjo, «Couvents de moniales dominicaines de la province de Teutonie», dans Marie-Anne Vannier (éd.), Encyclopédie des mystiques rhénans. D'Eckhart à Nicolas de Cues et leur réception, Paris, Cerf, 2011 (L'apogée de la théologie mystique de l'Église d'Occident), p. 308-316. 
Elsbeth Stagel, par «certaines pensées sublimes [...] dans la suave doctrine du saint Maître Eckhart», «considérée avec tant d'égards, comme il se doit $»^{4}-$, afin de les compiler dans des recueils en langue vulgaire. De la sorte, ces filles spirituelles prirent activement part, aux $\mathrm{XIV}^{\mathrm{e}}-\mathrm{XV}^{\mathrm{e}}$ siècles, non seulement à la diffusion, mais aussi à l'efflorescence d'une littérature d'édification conventuelle en moyen haut-allemand, nourrie du mysticisme rhénan 5 .

$\mathrm{La}$ «mystique rhénane»- mouvement de pensée propre à une région, à une époque et à une langue - «s'est cristallisée autour des trois figures eckhartienne, susienne et taulerienne d'un «maitre de lecture» (Lesemeister) qui serait aussi et d'abord un «maître de vie» (Lebemeister) $»^{6}$, et dont les enseignements trouvèrent ainsi un

4. Seuses Leben, c. 33, éd. K. Bihlmeyer (n. 1), p. 99, 10-13; trad. J. AnceletHustache (n. 1), p. 229. Voir Hans-Jochen Schiewer, «Uslesen. Das Weiterwirken mystischen Gedankenguts im Kontext dominikanischer Frauengemeinschaften », dans Walter Haug, Wolfram Schneider-Lastin (éd.), Deutsche Mystik im abendländischen Zusammenhang. Neu erschlossene Texte, neue methodische Ansätze, neue theoretische Konzepte, Tübingen, Niemeyer, 2000, p. 581-601, ici p. 595: «Entscheidend ist die Formulierung usgelesen uss der susssen lere dez heiligen maister Eghards. Offenbar spielt der Diener damit auf Lesefrüchte Elsbeth Stagels an, die sie - in welcher Form auch immer - aus Eckharts Schriften zusammengetragen hat». Voir également ci-dessus n. 1.

5. Voir Schiewer, «Uslesen... » (n. 4), où l'auteur - cherchant à savoir si, dans ce processus de transmission de la littérature spirituelle en langue vulgaire, les moniales, qui exercèrent la fonction de scribe, assumèrent également le rôle de compilator, c'est-à-dire remanier les textes et en composer des recueils d'extraits - développe l'hypothèse que «le procédé de la compilatio» (suivant le principe du uslesen) «caractérise le rapport productif à la littérature en langue vulgaire dans les couvents de dominicaines du XIV ${ }^{\mathrm{e}}$ siècle», lesquels n'apparaissent donc plus seulement comme un lieu de transmission, mais également comme un lieu de production de ce genre de littérature (p. 594-595 et p. 599 [Fazit]); car «si les moniales pouvaient écrire et lire, alors il ne peut y a voir d'objections sérieuses contre la supposition qu'elles se soient adonnées aussi au labor compilationis. En cela, elles pouvaient esquiver le continuel problème du rôle d'auctor et n'avaient besoin d'aucune justification supplémentaire pour leur activité littéraire. Elles ne modifiaient pas les textes, elles les arrangeaient seulement à nouveau frais » (p. 597). Voir également Andrea Syring, «Compilatio as a Method of Middle High German Literature Production », dans Jacqueline Hamesse, Beverly Mayne Kienzle, Debra L. Stoudt, Anne T. Thayer (éd.), Medieval Sermons and Society: Cloister, City, University, Louvain-La-Neuve, Brepols, 1998 (Textes et Études du Moyen Âge, 9), p. 117-143, où l'auteur montre certes que, du XIII ${ }^{\mathrm{e}}$ au XV $\mathrm{XV}^{\mathrm{e}}$ siècle, «la compilatio était une méthode répandue de production de la littérature monastique en langue vernaculaire» (p. 143), mais en ayant pris soin de préciser dès le début que «ce phénomène est décrit seulement pour un faible nombre de textes, spécialement ceux de Maître Eckhart» (p. 117).

6. Alain de Libera, «Mystique rhénane», dans Id., Claude Gauvard, Michel Zink (éd.), Dictionnaire du Moyen Âge, Paris, PUF, 2002, p. 959-962, ici p. 959960. 
fervent écho auprès de ces femmes studieuses qui, certes dépourvues d'une formation académique, mais manifestement réceptives à cette spiritualité nouvelle issue d'une prédication originale ${ }^{7}$, en produisirent diverses compilations dans les scriptoria des couvents, à travers lesquels se transmettaient les textes.

Au regard de ce processus médiéval de transmission de la parole des mystiques rhénans, les textes que l'on regroupe, depuis Adolf Spamer, sous le qualificatif de «traité-mosaïque» (Mosaiktraktat) ${ }^{8}$ méritent une attention particulière. Il s'agit là d'un conglomérat de plus ou moins courts fragments puisés intentionnellement dans les matériaux textuels à disposition (modus excerptionis), puis nouvellement agencés de manière thématiquement cohérente selon le principe de la compilation (modus compilationis), offrant une grande possibilité d'aménagement du texte. Le plus célèbre représentant de cette catégorie de traités est celui dont Carl J. Greith publia en 1861, sur la base de 2 manuscrits, une traduction en allemand moderne intitulée Das Lehrsystem der deutschen Mystik ${ }^{9}$. Or, depuis 1973 et l'édition du texte en moyen haut-allemand par Rosemary Cadigan ${ }^{10}$ - répondant au desideratum formulé par Josy

7. Il convient, avec Kurt Ruh, d'une part de souligner qu'«on n'admet plus aujourd'hui l'avis de Denifle selon lequel la mystique dominicaine est née de la rencontre entre le monde des Frères Prêcheurs et celui des moniales dominicaines, bien qu'il soit indéniable que cette rencontre se produisit au moment sociohistorique où naissait une mystique spécifiquement dominicaine»; qui plus est, d'autre part, «il est important de noter ici la place privilégiée qu'est appelé à occuper le sermon dans la diffusion de cette mystique». Mais on doit cependant minimiser la remarque selon laquelle «il est vrai que la prédication se prêtait particulièrement bien aux intentions de la cura monialium» et que «vue sous cet angle, la cura monialium serait à l'origine de la nouvelle prédication» (Kurt Ruh, Initiation à Maître Eckhart - Théologien, prédicateur, mystique, Paris-Fribourg, Cerf, 1997, p. 166). En effet, une récente étude de Loris Sturlese a fort bien montré qu'il s'agit davantage d'un «mythe», en tant que cette «hypothèse attend encore aujourd'hui d'être démontrée de manière convaincante» (Loris Sturlese, «Meister Eckhart und die Cura monialium: Kritische Anmerkungen zu einem forschungsgeschichtlichen Mythos», dans Andrés Quero-Sanchez, Georg Steer (éd.), Meister Eckharts Straßburger Jahrzehnt, Stuttgart, Kohlhammer, 2008 [Meister-Eckhart-Jahrbuch, 2], p. 11-16, ici p. 16).

8. Adolf Spamer, Über die Zersetzung und Vererbung in den deutschen Mystikertexten, Diss., Gießen, Hohmann, 1910; voir en particulier le premier chapitre du livre: «Zersetzung (Neubindung)», p. 18-28.

9. Carl J. Greith, Die deutsche Mystik im Prediger-Orden (von 1250-1350) nach ihren Grundlehren, Liedern und Lebensbildern, Freiburg im Breisgau, Herder, 1861, p. 96-203.

10. Rosemary Cadigan, The compilatio mystica (Greith's Traktat) in the original: an edition of Ms. C $108 \mathrm{~b}$ Zürich with reference to four other parallel manuscripts, Diss., University of North Carolina at Chapel Hill, 1973. 
Seitz, pour qui «la publication de Greith, du point de vue de la recherche actuelle, peut seulement servir comme palliatif ${ }^{11} \gg-$ ce compendium mysticum, rebaptisé Compilatio mystica, ne s'est toujours pas vu accorder toute l'attention que réclame sa valeur intrinsèque (telle une recherche plus détaillée de ses principes de cohérence $^{12}$, et par là-même de la technique du compilator $\left.^{13}\right)$.

Dès lors, ne pouvant prétendre, en l'espace d'un article, à une étude exhaustive sur l'apport de la Compilatio mystica comme tentative médiévale de systématisation de la mystique allemande et vecteur influent du discours doctrinal de ces principaux représentants, nous tâcherons seulement d'appuyer les raisons de son rattachement à l'école d'Eckhart.

\section{RéCEPTION DE LA COMPILATIO MYSTICA : \\ LE TÉMOIGNAGE DE QUELQUES NOTICES DANS CERTAINS MANUSCRITS}

La Compilatio mystica a été transmise par huit manuscrits: six donnant un traité complet ${ }^{14}$, un livrant une copie incomplète ${ }^{15}$, et un

11. Voir Josy Seitz, Der Traktat des 'Unbekannten dt. Mystikers' bei Greith, Diss., Rudolstadt, 1936, p. 114.

12. Voir B. Hasebrink, «Zersetzung» (n. 2), p. 79.

13. Voir Heidemarie Vogl, Der «Spiegel der Seele». Eine spätmittelalterliche mystisch-theologische Kompilation, Stuttgart, Kohlhammer, 2007 (MeisterEckhart-Jahrbuch. Beihefte, 2), p. 12. R. Cadigan (The Compilatio mystica [n. 10], p. XI) prévoyait pourtant que «dans les années à venir, la recherche sur ce traité, qui jusqu'à présent a largement été vouée à l'identification des textes parallèles, pourrait prendre une nouvelle direction. Puisque la Compilatio mystica est elle-même une création originale, le résultat d'un nouveau modèle appliqué à la documentation existante, il serait intéressant d'essayer de déterminer la méthode de compilation du scribe».

14. Sankt Gallen, Stiftsbibliothek, Cod. Sang. 1917 (vers 1400); Hamburg, Staats- und Universitätsbibliothek, Cod. theol. $18864^{\circ}$, p. 3-353; Konstanz, Bibliothek des Heinrich Suso-Gymnasiums, Cod. 37 [olim 27] (1484 [?]), Fasc. II, fol. 1r-102v; München, Bayerische Staatsbibliothek, Cgm 4373 (milieu du $\mathrm{XV}^{\mathrm{e}}$ s.), fol. 2r-146v et Cgm 5233 (milieu ou dernier quart du Xv ${ }^{\mathrm{e}}$ s.), fol. 1r-104v; Zürich, Zentralbibliothek, Cod. C 108b (début du $\mathrm{XV}^{\mathrm{e}} \mathrm{s}$.). Mentionné par Volker Honemann sur la base d'une indication de Werner Williams-Krapp ( «Lehrsystem der deutschen Mystik», dans Verfasserlexikon, $2^{\mathrm{e}}$ éd., t. 5, Berlin, De Gruyter, 1985, col. 676-678), le manuscrit de Constance demeurait inconnu de Cadigan et n'a donc pas été pris en considération dans son édition. N'ayant malheureusement pas eu l'occasion de consulter ce manuscrit - lequel, à notre connaissance, n'a encore fait l'objet d'aucune description -, nous n'avons pu comparer cette version avec le texte établit par Cadigan.

15. Augsburg, Universitätsbibliothek, Cod. Öttingen-Wallerstein III $1.8^{\circ} 42$ $\left(\mathrm{XV}^{\mathrm{e}}\right.$ siècle), fol. 3r-38r. Concernant cette version incomplète, non retenue par 
contenant seulement des fragments qui constituent le plus ancien témoin répertorié, daté de la fin du XIV ${ }^{\mathrm{e}}$ siècle $^{16}$. L'étude comparative de la tradition manuscrite a révélé deux versions différentes de la compilation quant au volume du texte et à l'ordonnance thématique ${ }^{17}$ : «une primitive [?], mal ordonnée (manuscrits Saint-Gall et Cgm 5233), et une plus clairement structurée, un peu plus récente [?] (manuscrits restants) $\gg^{18}$. À l'exception du manuscrit d'Hambourg, tous les autres ont été copiés dans des couvents de dominicaines du sud-ouest de l'Alémanie, confirmant la réception et la circulation remarquables des textes eckhartiens dans cette région ${ }^{19}$.

Quant au codex retenu par Cadigan comme lead manuscript de son édition - lequel contient exclusivement la Compilatio mystica on dispose de peu d'informations sur sa provenance. Selon Leo Cunibert Mohlberg, il aurait vraisemblablement été copié dans le couvent des dominicaines de Töss, localisé à proximité de la ville de Winterthur dans le canton de Zürich ${ }^{20}$. En 1772, le pasteur et

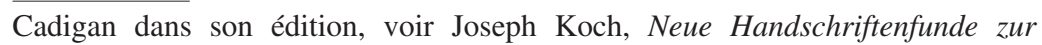
Überlieferung der deutschen Werke Meister Eckharts und seiner Schule, StuttgartBerlin, Kohlhammer, 1940 (Meister Eckhart, Die deutschen und lateinischen Werke. Untersuchungen, 1), p. 124-127; voir notamment p. 127, où Koch signale qu'il a «renoncé à identifier en détail l'origine des différents éléments du traité mosaïque, excepté quelques passages ».

16. Stuttgart, Württembergische Landesbibliothek, Cod. theol. et philos. $4^{\circ} 219$, Einband (fin du XIV ${ }^{\mathrm{e}}$ s.). Sur la datation de ce manuscrit, voir Alban Dold, «Die Geschichte eines Bucheinbandes und die Ergebnisse seiner Untersuchung», Zeitschrift für Schweizerische Kirchengeschichte, 44 (1990), p. 241-258, ici p. 243-246 et 257; ainsi que Judith Theben, Die mystische Lyrik des 14. und 15. Jahrhunderts: Untersuchungen - Texte - Repertorium, Berlin-New York, De Gruyter, 2010 (Kulturtopographie des alemanischen Raums, 2), p. 179.

17. Sur la relation entre les différents manuscrits complets (excepté celui de Constance), voir R. Cadigan, The Compilatio mystica (n. 10), p. LXV-LXXIX.

18. V. Honemann, «Lehrsystem» (n. 14), col. 676.

19. Voir, par exemple, Adolf Spamer, «Zur Überlieferung der Pfeifferschen Eckharttexte», Beiträge zur Geschichte der deutschen Sprache und Literatur, 34 (1909), p. 307-402, ici p. 344, qui, sur la base de l'ensemble des manuscrits connus jusqu'alors, établit que la transmission des sermons eckhartiens se répartit géographiquement en trois groupes, dont «le premier et le plus grand groupe est celui d'Allemagne du sud et spécialement du sud-ouest, ayant pour centre Strasbourg [...]. Outre Strasbourg, entre notamment Bâle en ligne de compte. De plus, en font partie l'ensemble de la Suisse, la Souabe (par exemple le couvent de Weingarten), de même qu'aussi la région bavaroise».

20. Voir Leo Cunibert Mohlberg, Katalog der Handschriften der Zentralbibliothek Zürich, I: Mittelalterliche Handschriften, Zürich, Zentralbibliothek Zürich, 1952, Nr. 140, p. 56-57. Toutefois, Marie-Claire DänikerGysin (Geschichte des Dominikanerklosters Töss: 1233-1525, Winterthur, 
historien zurichois Johann Konrad Füßli redécouvrit ce codex, le qualifiant alors de «Livre mystique sur Dieu, l'âme et l'homme, compilé à partir de Denys et d'autres Mystiques ${ }^{21} \gg$. Cette description assez générale - qui témoigne de l'apparition du terme «mystique» employé comme substantif, et non plus seulement comme adjectif (suivant l'acception médiévale) ${ }^{22}$ - est complétée par une notice ramassant successivement le titre, l'auteur, le contexte et la teneur de ce texte:

Le titre de ce livre est: Dialogus veritatis cum discipulo suo ou Gespräch der Wahrheit mit ihrem Schüler [Dialogue de la Vérité avec son disciple]. L'auteur est Henri Suso, tantôt dit Amandus, moine dominicain, lequel est né à Constance en l'an du Seigneur 1300 et mort à Ulm en 1365. Il a souvent séjourné dans les couvents de Töss et d'Oetenbach à Zurich, là même où il avait ses disciples ou, ainsi dites, pénitentes. Elisabetha Stahl [Elsbeth Stagel], qui est comptée parmi les saintes, écrivit sa biographie. Or Suso était un mystique et ce livre est tout entier mystique, un authentique modèle de théologie mystique ${ }^{23}$.

Stadtbibliothek Winterthur, 1958, p. 96) souligne qu'aucun indice ne permet de confirmer cette provenance. Qui plus est, la note apposée sur le deuxième de couverture (par une seconde main inconnue): Dz Buech gehört in dz buech Ampt est considérée comme une inscription typique d'appartenance à la bibliothèque du couvent de dominicaines Sainte-Catherine d'Augsbourg.

21. «Liber mysticus de Deo, anima et homine ex Dionisio caeterisque Mysticis collectus» cité d'après Otto Karrer, "Aus einer mittelalterlichen Mystikerhandschrift», Wiedergeburt. Jahrbuch des Verbandes der RenaissanceGesellschaften, 8 (1929/30), p. 7-15, ici p. 7.

22. Alain de Libera, Penser au Moyen Âge, Paris, Seuil, 1991, p. 301-302: "Le terme "mystique" est équivoque, c'est à la fois un substantif et un adjectif. Reconduit à son acception médiévale, il redevient univoque [...]. Il faut le redire: au Moyen Âge, "mystique" est un adjectif qui s'emploie exclusivement pour qualifier un type de théologie. Aux yeux d'un esprit médiéval, il n'y a donc pas plus d'"états" que d'hommes ou de femmes "mystiques". [...] Si donc le Moyen Âge ne connaît pas d'autre theologia mystica que celle de Denys, où seul Dieu est, au sens propre du terme, "mystique", c'est-à-dire caché - la théologie mystique étant une méthode, un chemin d'accès à Dieu caché en Dieu lui-même -, la notion de "mystique médiévale", entendue comme désignant un ensemble de comportements singuliers ou un type particulier d'individus doit être considéré comme non descriptive: c'est une catégorie de l'historiographie; ce n'est pas un objet de l'Histoire».

23. "Inscriptio huius Libri est: Dialogus veritatis cum discipulo suo oder Gespräch der wahrheit mit ihrem Schüler. Autor est Henricus Suso, alias Amandus dictus, Monachus dominicanus, qui Constantiae natus fuit A. D. 1300 et Ulmae mortuus 1365. Saepe in coenobiis Tösano et Oetenbacensi Thurici moratus est, ibique suas habuit discipulas seu poenitentes ita dictas. Elisabetha stahl, quae in sanctis numeratur, eius vitam scripsit. Fuit autem Suso mysticus et liber hic totus est mysticus, Theologiae mysticae genuinum Exemplar», cité d'après Cadigan, The Compilatio mystica (n. 10), p. XXI. 
Füßli était donc persuadé qu'il s'agissait là d'un texte de Suso, dont il possédait somme toute une bonne connaissance de l'œuvre ${ }^{24}$. Bien qu'erronée, cette attribution - qui sera partiellement reconduite par Greith ${ }^{25}$ - avait néanmoins le mérite de situer ce traité dans le giron de la mystique rhénane, puis de mettre en exergue sa valeur exemplaire en tant qu' Hauptsystematum der mystischen Theologie ${ }^{26}$.

Consultons maintenant le plus récent des manuscrits recensés par Cadigan, celui de Hambourg, qui renferme «la meilleure et la plus correcte copie ${ }^{27} »$, réalisée en 1614 par Daniel Sudermann - poète protestant allemand, adepte de la spiritualité mystique et collectionneur de manuscrits ${ }^{28}$. Celui-ci rapporte au dos de la seconde page comment «parmi de nombreux autres livres et textes d'un noble couvent de vierges de Cologne (à savoir celui de Sainte-Gertrude, dans lequel a demeuré Tauler)», il s'est procuré «subrepticement et de manière confidentielle» ce manuscrit «par quelqu'un de sa lignée et proche cousine $»^{29}$ (le colophon de la page 353 nous renseigne sur la date de cet alten exemplar colonais à partir duquel a été copié - probablement à Strasbourg - le manuscrit de Hambourg: Anno $\left.1475^{30}\right)$. Non seulement collectionneur d'envergure mais aussi

24. Voir Seitz, Der Traktat des 'Unbekannten dt. Mystikers' (n. 11), p. 49-50.

25. Voir C. J. Greith, Die deutsche Mystik im Prediger-Orden (n. 9), p. 81: «Mit Heinrich Suso hängt der ungenannte deutsche Mystiker zusammen». Mohlberg (Katalog der Handschriften der Zentralbibliothek Zürich [n. 20], p. 56) catalogua quant à lui ce texte comme «traité anonyme (de l'école de Suso) sur la théologie mystique».

26. J. Seitz, Der Traktat des 'Unbekannten dt. Mystikers' (n. 11), p. 113.

27. Voir R. Cadigan, The Compilatio mystica (n. 10), p. xxxv.

28. Voir Ingeborg Degenhardt, Studien zum Wandel des Eckhartbildes, Leyde, Brill, 1967 (Studien zur Problemgeschichte der antiken und mittelalterlichen Philosophie, 3), chap. V («Daniel Sudermann - ein Sammler der Eckhartschriften im Barock»), p. 90-102.

29. «Wie dann dieses neben vielen andern büchern vnd schrifften auß einem adelichen Jungfrawen Kloster zu Cölln (halt zu S. Gertruden, in welchem Taulerus gewesen) von einer seines geschlecht $\beta$ vnd naher basen D. S. heimlich v. vertrawlicher weiß erhalten vnd bekommen», cité d'après R. Cadigan, The Compilatio mystica (n. 10), p. XxIx. Le catalogue de la volumineuse bibliothèque du maire francfortois Zacharias Conrad von Uffenbach (1683-1734), dans laquelle était auparavant conservé le manuscrit de Hambourg, mentionnait également l'utilisation par Sudermann de manuscrits colonais: «Libri aliquot vet. mystici, quos D. J. H. Sudermannus e vet. codi. monasterii cujusdam Coloniensis descripsit et ab interitu vindicavit. In fine accedunt duo scripta recentiora» (Bibliothecae Uffenbachianae Universalis tomus III, Francfort, Andreae \& Hort, 1730, p. 721, Nr. 56).

30. «Vnd ist dann dieses von einem alten exemplar, so wie am end zu finden, anno 1475 geschrieben worden, abgeschrieben», cité d'après R. Cadigan, The 
lecteur attentif de manuscrits, Sudermann ambitionnait notamment d'en identifier les textes transmis anonymement, de sorte qu'il en vient certes ici à présenter d'abord la Compilatio mystica comme «un noble livre ou extraits des tous meilleurs maîtres spirituels» (sous la forme d' «un dialogue entre un maître et son disciple»), mais en spécifiant aussitôt que lesdits extraits («ordonnés pour le bien de tous vrais imitateurs du Christ») sont «singulièrement et assurément presque tous à trouver dans les écrits du Nobilis Magistri Eckhardi ${ }^{31}$. Sur ce point important, Sudermann ajoute une remarque complémentaire dans la marge:

Il semble certain que ce livre entier ait puisé aux écrits de cet homme hautement illuminé par Dieu. Ce qu'il y a de mieux a donc été découvert parmi les livres de Tauler. Mais, par la langue, il est bien à croire que c'est antérieur au vécu de Tauler, dont céans il n'est jamais fait état. Le vocabulaire et le style dans le livre Sur [l'imitation de] la vie pauvre du Christ sont très similaires à celui-ci, si bien que les deux sont donc de M[aître] Eckhart. Pourtant, les catholiques ont attribué tous ses sermons et livres à Tauler ${ }^{32}$.

À la page suivante, Sudermann souligne que l'issue du procès en inquisition contre Eckhart eut pour répercussion que ce dernier est à l'époque inconnu «du monde entier et des érudits de la Bible», mais que «cependant, en raison de son divin enseignement, nombre

Compilatio mystica (n. 10), p. xxIx. Même s'il n'existe malheureusement aucune trace de ce codex, «demeure d'un grand intérêt le fait que justement la ville de Cologne et justement le couvent Sainte-Gertrude de Cologne livre encore une copie du traité et prouve en cela que dans cette ville classique de la mystique allemande, où les grands maîtres ont œuvré et fréquenté personnellement ce couvent de femmes, le traité fut encore estimé un siècle plus tard en raison d'un fort sens de la tradition » (Seitz, Der Traktat des 'Unbekannten dt. Mystikers' [n. 11], p. 11). Voir Jutta Prieur, Das Kölner Dominikanerinnenkloster St. Gertrud am Neumarkt, Diss., Cologne, DME, 1983 (Kölner Schriften zu Geschichte und Kultur, 3), p. 434-437 et 474.

31. «Ein edell buoch oder Außzueg der aller bessten geistlichen Lehren, so von den Alt Vättern, sonderlich aber und fast gantz durchauß in Nobilis Magistri Eckhardi schrifften zu finden, in eine ordnung (allen wahren Christi Nachfolgeren zu gutem) gebracht worden. Ist ein Dialogus oder gespräch von einem Meister und seinem Jünger », cité d'après J. Seitz, Der Traktat des 'Unbekannten dt. Mystikers' (n. 11), p. 12.

32. «Es scheint gewiß dz diß gantz buch auß dieses von Gott hoch-erleuchten manns schriften genomen. Ist daz besste so unter Taulers büchern gefunden worden. Es ist aber von der sprach wol abzunehmen daß es älter ist alß Taulerus gelebet, dessen hier innen nie gedacht wird. Die wörter v[nd] der stylus im buch von armen leben Christi gleichen diesem sehr, mögen also wohl beÿde M. Eckhards sein. Die Catholischen aber schrieben alle seine predigen v[nd] bücher Taulero zue. D. S. », cité d'après R. Cadigan, The Compilatio mystica (n. 10), p. xxx. 
de ses livres et écrits sont parfois trouvés dans des couvents, qui les dissimulent à tout le monde et ne laissent y accéder l'homme ordinaire $»^{33}$. Les annotations de Sudermann sont pour lors très instructives, car afin de légitimer son constat d'une indéniable paternité eckhartienne de la Compilatio mystica, il entend faire valoir deux facteurs-clé d'une transmission médiate et clandestine de sermons et traités allemands d'Eckhart suite à sa condamnation (et à la censure consécutive): d'une part, leur incorporation à l'œuvre de son disciple Jean Tauler, auquel ils furent donc faussement attribués; d'autre part, leur conservation en secret dans les couvents de dominicaines, qui tentèrent d'assurer ainsi la pérennité de l'enseignement du Maître rhénan - par exemple, comme ici, en dissimulant quantité de fragments au sein d'un traité-mosaïque. Pour Sudermann toutefois, et c'est là le plus intéressant, de tels procédés ne sauraient malgré tout faire oublier les caractéristiques propres au discours d'Eckhart - tant sur le fond que sur la forme -, puisqu'il apparaissait possible à cet adepte de la mystique rhénane d'identifier (par comparaison) les passages typiquement eckhartiens ${ }^{34}$ et, par conséquent, de les réattribuer (mais sans arguments réellement fiable ${ }^{35}$ ) à celui qui « fut le devancier de Tauler et quasi le plus illuminé par Dieu parmi tous les anciens maîtres $»^{36}$.

33. «Magister Eckhardus ist des Tauleri vorfahr vnd fast der aller erleüchteste von Gott vnder den alten lehrern gewesen, Anno 1300 vnd etliche hernach, von den Inquisitoren zu Heÿdelberg verbrännt worden, daher von allen welt vnd schrifft-gelehrten noch vnbekannt, doch von wegen seiner Göttlichen lehre werden viel seiner bücher vnd schrifften in klösteren hien vnd wieder gefunden, welche si vor Jedermann verbergen vnd nicht vnter den gemeinen man kommen lassen, biß etwan ein kloster zerstöret oder mit gewalt eingenommen wirdt, oder sie Gott selbß herfürschaffet. D. S.», cité d'après R. Cadigan, The Compilatio mystica (n. 10), p. XXX-XXXI. Sudermann rapporte donc ici qu'Eckhart «et de nombreux à sa suite ont été brûlés par les inquisiteurs à Heidelberg». Selon I. Degenhardt (Studien zum Wandel des Eckhartbildes [n. 28], p. 94), Sudermann amalgame vraisemblablement la condamnation des écrits d'Eckhart par les théologiens d'Heidelberg (telle que le rapporte Johannes Trithemius dans son Catalogus scriptorum ecclesiasticorum, rédigé en 1494) et quelque retentissante mise au bûcher d'hérétiques dans cette même ville.

34. À tort ou à raison, Sudermann identifia ainsi explicitement au moins six passages de la Compilatio mystica, ce dont témoignent certaines gloses marginales dans le manuscrit de Hambourg; voir fol. 40r' : «M. Eckh.»; fol. 113v $\mathrm{v}^{5}$ : «M.

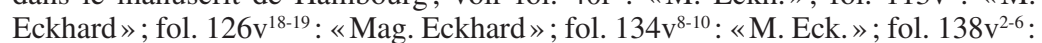
«Diß ist ein hohe lehr v. eigentlich M. Eckhards»; fol. $150 v^{6-8}:$ M. Eckhard».

35. Voir I. Degenhardt, Studien zum Wandel des Eckhartbildes (n. 28), p. 93.

36. Voir ci-dessus, n. 35. Deux siècles plus tard, le philosophe allemand Franz von Baader soutiendra de manière comparable qu'Eckhart est «der erleuchteste 
Les supputations de Sudermann, puisqu'elles ne sont pas fondées sur un examen critique du texte de la Compilatio mystica, nous incitent dès lors, à l'appui de l'édition de Cadigan, à y cerner schématiquement les principaux aspects d'une telle spécificité, tant sys-thématique que linguistique, du discours eckhartien et, $a$ fortiori, eckhartiste.

\section{UN LEHRSYSTEM DE LA MYSTIQUE (MOYEN-HAUT) ALLEMANDE}

\section{Une cohérence sys-thématique}

L'étroite observance eckhartienne de la Compilatio mystica se laisse d'abord justifier par le fait qu' on y retrouve en substance les quatre points du programme de prédication de Maître Eckhart ${ }^{37}$, tels qu'énoncés au début du Sermon allemand $53^{38}$ :

Quand je prêche, j'ai coutume de parler du détachement et de dire que l'homme doit être dépris de lui-même et de toutes choses. En second lieu, que l'on doit être réintroduit dans le Bien simple qui est Dieu. En troisième lieu, que l'on se souvienne de la grande noblesse que Dieu a mise dans l'âme afin que l'homme parvienne ainsi merveilleusement jusqu'à Dieu. En quatrième lieu, je parle de la pureté de la nature divine - de quelle clarté est la nature divine, c'est inexprimable. Dieu est une Parole, une parole inexprimée ${ }^{39}$.

aller Theologen des Mittelalters» (Sämmtliche Werke, vol. XIV, Leipzig, Herrmann Bethmann, 1851, p. 93), ce qui l'amènera par ailleurs à qualifier le Maître rhénan de «Centralgeist der Mystik des Mittelalters» (Sämmtliche Werke, vol. XV, Leipzig, Herrmann Bethmann, 1857, p. 457).

37. Voir J. Seitz, Der Traktat des 'Unbekannten dt. Mystikers' (n. 11), p. 101.

38. Voir, entre autres, Joseph Koch, «Meister Eckhart. Versuch eines Gesamtbildes», dans Id. (éd.), Kleine Schriften, I, Rome, Edizioni di Storia e Letteratura, 1973 (Storia e Letteratura, Raccolta di Studi e Testi, 127), p. 201238, ici p. 226-238 (V); et Alois Maria Haas, «Meister Eckharts geistliches Predigtprogramm», Freiburger Zeitschrift für Philosophie, 29 (1982), p. 192-209.

39. Maître Eckhart, Predigt 53, éd. Josef Quint, dans Die deutschen Werke $[D W]$, II, Stuttgart, Kohlhammer, 1971, p. 528, 5-529, 2: «Swenne ich predige, sô pflige ich ze sprechenne von abegescheidenheit und daz der mensche ledic werde sîn selbes und aller dinge. Ze dem andern mâle, daz man wider îngebildet werde in daz einvaltige guot, daz got ist. Ze dem dritten mâle, daz man gedenke der grôzen edelkeit, die got an die sêle hât geleget, daz der mensche dâ mite kome in ein wunder ze gote. Ze dem vierden mâle von götlîcher natûre lûterkeit - waz klârheit an götlîcher natûre sî, daz ist unsprechelich. Got ist ein wort, ein ungesprochen wort». Le compilateur avait vraisemblablement connaissance de ce sermon, puisqu'il en reprend deux fragments dans le traité; voir Compilatio mystica, éd. R. Cadigan (n. 10), p. 224, 15-22 et p. 297, 19-298, 6. 
Ces quatre thèmes fondamentaux ${ }^{40}$, bien qu'expressément assimilés par la Compilatio mystica, n'y apparaissent toutefois qu'en lignes de fond, c'est-à-dire sans revêtir dans cet ordre une rigoureuse valeur systématique et sans dicter ainsi le plan du traité. Celui-ci s'organise de fait autour du thème biblique cité en incipit: «Maintenant écoute et apprends comment la Sainte Trinité a dit dans le premier livre de Moïse: Faisons un homme à notre image et à notre ressemblance (Gn. 1, 26). Et prends-en bonne note, car c'est un fondement de la connaissance divine $»^{41}$. Ce verset du livre de la Genèse fournit le leitmotiv théologique du traité-mosaïque, et par conséquent le principe de sélection des matériaux qui composent cet édifice textuel ${ }^{42}$. Or, suivant Heribert Fischer, qui s'employa à déterminer les idées fondamentales se dégageant de la prédication d'Eckhart en langue vernaculaire, une vue d'ensemble des sermons allemands pourrait justement retenir comme point de départ d'une approche théologique le texte de Gn. 1, 2643, puisqu'il engage l'affirmation ultime de Dieu quant à la noblesse de l'âme humaine ${ }^{44}$. Sous-tendue par une volonté didactique d'éclairer la portée de ce verset, la Compilatio mystica se structure en trois sections: la première, développant une théorie de la connaissance, porte sur l'âme et ses puissances comme image et ressemblance de La Trinité; la seconde, relevant de la mystique spéculative, traite du Dieu trinitaire et de la vie divine dans l'âme; la troisième, d'orientation plus ascétique, décrit enfin le chemin vers Dieu et l'union avec lui ${ }^{45}$. Cet enchaînement trahit clairement une intention sys-thématique de la part du compilateur, c'est-à-dire l'objectif d'ériger avec concision un système cohérent (et plus ou moins abouti) décryptant le raccordement de divers enseignements et

40. Les trois premiers, selon K. Ruh, «constituent le véritable réceptacle de la spiritualité mystique du maître», tandis que le dernier «représente pour sa part le lieu d'exposition de sa théologie spéculative» (Initiation à Maître Eckhart [n. 7], p. 208).

41. Compilatio mystica, éd. R. Cadigan (n. 10), p. 1, 1-9: «Nun hœre vnd vernieme, wie die hailig drivaltikeit gesprochen hat jn dem ersten buoche moisy, also: "Wir sœllent machen ainen menschen nach vnserm bilde vnd gelichnússe". Vnd dis merke eben, wan es ist ain grunde gœtlicher bekantnússe.»

42. Voir J. Seitz, Der Traktat des 'Unbekannten dt. Mystikers' (n. 11), p. 95.

43. Voir Heribert Fischer, «Grundgedanken der deutschen Predigten», dans Udo Maria Nix, Raphaël Öchslin (éd.), Meister Eckhart der Prediger: Festschrift zum Eckhart-Gedenkjahr, Freiburg, Herder, 1960, p. 25-72, p. 29.

44. Ibid., p. 33

45. Voir J. Seitz, Der Traktat des 'Unbekannten dt. Mystikers' (n. 11), p. 103113. 
niveaux de discours de la théologie mystique allemande médiévale, au profit d'un agencement thématique. Il se dégage du coup une certaine impression d'homogénéité de ce Lehrsystem, permettant non seulement d'en suivre la progression argumentative, mais également d'en saisir l'enjeu doctrinal et spirituel essentiel: conduire l'âme humaine, en raison de sa noblesse (edelkeit) ${ }^{46}$, vers l'union mystique à Dieu ${ }^{47}$ et la naissance de celui-ci dans le fond de l'âme ${ }^{48}$, en exhortant l'homme au détachement (abgeschaidenheit) ${ }^{49}$ et à l'abandon (gelassenheit) $)^{50}$, qui constituent «les deux maîtres mots de la mystique allemande ${ }^{51} »$.

46. Voir par exemple Compilatio mystica, éd. R. Cadigan (n. 10), p. 153, 7-13: «Wan die sele, die ist von got so edel vnd so luter geschaffen»; p. 173,923: «Vnd hier vmbe wene nun die sele [...] sich selber anschouwet in ir edelkeit, wie sie got von nihte ze ichte geschaffen hat».

47. Voir par exemple ibid., p. 307, 8-11: «Vnd wer nit kumet in die ainunge des gaistes mit gotte, der enist nit ain rechter gaistlicher mensche».

48. Voir par exemple $i b i d .$, p. 300, 20-302, 1: «Vnd hier vmbe sprichet maister eckhart: Vnd wende ir wissen, wenne der himelsche vatter gebirt sinen sun in der sele, [...], da wirt der sun geboren als in dem himelschen vatter. Wan ain aigenschaft der gœtlichen geburt ist, das si alwege beschiht mit núwem lieht, wan si bringet alwegent grose lieht in die sele. Wan gottes geberen in der sele ist nit anders, wan dz sich got in der sele zœget vnd offenbaret jn ainer núwen bekantnúst vnd mit ainer núwen wise. Wan in dieser geburt er-gússet sich got in die sele mit lieht also, $\mathrm{dz} \mathrm{dz}$ lieht also grose wirt in dem wesen vnd in dem grunde der sele».

49. Voir par exemple ibid., p. 120,1-7: «vnd das mainet, dz dú sele ab schaiden sol mit vernunftikeit alles, das man got zuo gelegen mag an gedenken oder in verstavn»; p. 130, 10-15: «aber gœtliche warheit ist úber bilde jn ainer blosen abgeschaidenheit von allen bilden, da nieman von gereden kann noch mag»; p. 600, 9-13: «Vnd also nach dem allernechsten so muß man abscheiden all liplich vnd gaistlich materien, vnd das alle bilde vnd formen, die sich offenbarent».

50. Voir par exemple ibid., p. 329, 20-330, 3: «Vnd alsus an allen disen vnd menigen andern, die man geschriben mœhti, ensol man sich nit lassen, denne in ain diemuetig entsinken in rechter gelassenheit»; p. 338, 17-339, 11: «Vnd als denne der mensche stavt in rechter lediger gelassenheit [...], vnd denne so wirt der mensche alzemale vf gerich[t]et vnd belibet vngehindert vnd mag sich inkeren in sinen ursprunge vnd in sin vngeschaffenheit, da er ewenklichen gewesen ist»; p. 600, 5-9: «Vnd also muß ain gelassen mensch enbildet werden von der creature vnd gebildet werden mit cristo vnd v́ber bildet werden in der gothait». Ce dernier passage, emprunté au chapitre 49 de la Vita d'Henri Suso (mais seulement compilé dans la première version du traité), systématise à lui seul les trois acceptions de la gelassenheit développées par la mystique rhénane. Notons alors qu'il s'agit là d'une paraphrase accentuée d'un passage du Sermon allemand 40 d'Eckhart ( $D W$, II, p. 278, 4-6) et, par suite, d'une interprétation susienne du ternaire eckhartien entbilden-înbilden-überbilden, lequel structure fondamentalement toute l'anthropologie spirituelle du Maître rhénan.

51. Alain de Libera, "Sérénité et détachement chez Maître Eckhart», dans Charles Gagnebin, Daniel Schulthess, Gerhard Seel (éd.), Le dépassement de 


\section{Une langue et un style caractéristiques}

La provenance intellectuelle de l'enseignement systématisé par la Compilatio mystica se détermine donc aussi à l'aune de sa spécificité conceptuelle, empreinte de la pratique linguistique novatrice d'un Maître Eckhart, «professionnellement latinophone», et dont les sermons en moyen haut-allemand, selon Alain de Libera, «effectuent le premier passage de la langue de l'École à la langue vulgaire ${ }^{52} »$. Alain de Libera distingue ainsi cinq sortes de cas illustrant ce transfert du latin scolastique au vernaculaire $^{53}$, tous aisément repérables dans la Compilatio mystica. Celle-ci offre de fait un large terrain d'application générale aux diverses études consacrées à la Begriffssprache d'Eckhart ${ }^{54}$, considérée habituellement comme étant à l'origine de la formation du langage philosophique allemand ${ }^{55}$, notamment

soi dans la pensée philosophique. Actes du colloque des 19 et 20 octobre 1990 pour les soixante-dix ans de Fernand Brunner, Neuchâtel, La Bocannière, 1994, p. 27-53, ici p. 40. Voir aussi Markus Enders, Gelassenheit und Abgeschiedenheit - Studien zur Deutschen Mystik, Hambourg, Kovač, 2008.

52. A. de Libera, «Sermo mysticus. La transposition du vocabulaire scolastique dans la mystique allemande du $\mathrm{XIV}^{\mathrm{e}}$ siècle», Rue Descartes 14: De l'intraduisible en philosophie (1995), p. 41-73, ici p. 44. On signalera qu'il est fait seulement une fois référence à la langue latine dans la Compilatio mystica, au fol. $60 \mathrm{v}^{8-13}$ (moyennant un extrait du Sermon allemand 44 d'Eckhart): «Et c'est pourquoi le mot erat appartient le plus proprement à Dieu, car dans la langue latine (in laitinischer zungen) il n'y a donc aucun mot qui soit plus propre à Dieu qu'erat».

53. Voir ibid., p. 51: «1. les traductions eckhartiennes du latin qui ne posent pas de problèmes linguistiques, mais qui soit présentent les mêmes équivoques que les termes traduits, soit reflètent l'inconsistance du complexe théorique auxquels ces termes appartiennent, ou l'inconsistance de la lecture qu'en donne Eckhart lui-même $[\ldots] ; 2$. Les néologismes qui fonctionnent comme des échangeurs entre divers termes ou champs, qu'ils soient non saturés $[\ldots]$ ou saturés $[\ldots] ; 3$. Les intraduisibles, qui imposent des néologismes ou des périphrases au traducteur français $[\ldots]$; 4. Les complexes de termes qui s'inscrivent comme un moment d'indécision dans l'histoire de l'être [...]; 5. Les termes qui, à l'époque moderne, ont fait l'objet d'un supplément de germanisation à partir d'intuitions néohégéliennes ou idéalistes spéculatives ».

54. Voir Niklaus Largier, Bibliographie zu Meister Eckhart, Freiburg, Universitätsverlag Freiburg, 1989 (Dokimion, 9), p. 100-107 [«IX. Sprache und Form»]. Rappelons toutefois ici, avec Seitz (Der Traktat des 'Unbekannten dt. Mystikers' [n. 11], p. 91), que «le traité, en tant que compilation n'offre aucune possibilité de complément aux travaux existants».

55. Voir Hermann Kunisch, «Die mittelalterliche Mystik und die deutsche Sprache. Ein Grundriß», Literaturwissenschaftliches Jahrbuch, Neue Folge 6 
à travers la création (résultant d'un "geste traducteur $»^{56}$ ) d'une vaste gamme de préfixations et suffixations verbales et nominales. Dans la Compilatio mystica, on relèvera entre autres l'abondance de substantifs abstraits formés à l'aide des suffixes -keit/-heit (par exemple ainikeit, vernúnftikeit, gaistlicheit, i[h/s]tikeit, gelassenheit, abgeschaidenheit) et nomina actionis en -unge (par exemple begriffunge, bildunge, anschouwunge, ainunge), ou encore la récurrence des superlatifs composés avec le préfixe v́ber- (ainsi v́ber-wesenlich, v́ber-natúrlich, v́ber-verstentlich, v́ber-redelich), dont l'emploi «apporte une solennité particulière au discours mystique et rend le sens d'inaccessibilité et de perfection de tout ce qui est transcendant $\gg^{57}$. L'expression de cette transcendance est en outre sauvegardée par un recours systématique au langage négatif d'origine dionysienne, tant prisé par Maître Eckhart et ses disciples en tant qu'il répond à une insuffisance inhérente au langage affirmatif pour décrire l'ineffabilité de Dieu, lequel est alors appréhendé comme «un non-être» (ain nit wesen) et «un néant» (ain niht) signifiant «je-ne-sais-quoi» (naiswaz) d' «inconcevable» (vnbegriffenlich), c'est-à-dire «l'Être sans mode» (wiselos) et, par conséquent, «innommable» (namelos). Ce discours apophatique transmis par la Compilatio mystica, laquelle véhicule donc tacitement la question du statut même du langage mystique, se caractérise par l'emploi de quelques images typiques (par exemple celle de la ténèbre: in dem verborgnen dúnsternússe des vnbekanten gottes $^{58}$, in dem dúnsternússe des nihtes ${ }^{59}$ ), de tournures paradoxales (ainsi Da sihet si got vnd durch sichet sin nicht; da sol si got bekennen vnd ensol in doch niemer ze grunde bekennen; da sol si got begriffen vnd ensol in doch vnbegriffen ${ }^{60}$ ),

(1965), p. 37-90; Christopher Jon Wells, Deutsch: Eine Sprachgeschichte bis 1945, Tubingen, Niemeyer, 1990, p. 144.

56. A. de Libera, «Sermo mysticus $»($ n. 49), p. 41.

57. D. Bremer Buono, «Le langage de la mystique dans l'œuvre allemande de Maître Eckhart», dans Emilie Zum Brunn (éd.), Voici Maître Eckhart, Grenoble, Jérôme Millon, 1994, p. 243-268, ici p. 246.

58. Compilatio mystica, éd. R. Cadigan (n. 10), p. 234, 1-3; trad.: «dans la ténèbre cachée du Dieu inconnu ».

59. Ibid., p. 263, 12-13; trad.: «dans la ténèbre du néant».

60. Ibid., p. 272, 19-24; trad.: «Alors elle [1'âme] voit Dieu et [cependant] ne Le voit pas à travers Lui; alors elle doit connaître Dieu et cependant ne doit jamais Le connaître en Son fond; alors elle doit comprendre Dieu et cependant ne doit pas Le comprendre [comme Il se comprend Lui-même]». 
accentuées par certains oxymorons (comme $d z$ die sele mit ainem versinkende nit-suochen den suochet den grundelosen grunt der gotheit $\left.t^{61}\right)$. On relèvera de surcroît la présence massive du concept grund $^{62}$ - appliqué tantôt à l'Être divin (grund gætliches wesen), tantôt à l'âme (grund der sele) en son essence intellective -, accompagné de l'épithète grundelos - servant à exprimer métaphoriquement l'insondabilité du mystère divin (par exemple jn dem grundelosen mere der gotheit $t^{63}$, jn dem grundelosen brunen getlicher nature $\left.{ }^{64}\right)$. Transmettant de la sorte quantité de notions et expressions appartenant en propre à la terminologie eckhartienne et représentatives - par extension - du lexique de la mystique rhénane (telles que les créations linguistiques vsflússe, vs-bruche, in-druk, v́ber-flússikeit, vernichtikait, vergaisten, vergœten, gotformig), la Compilatio mystica témoigne remarquablement de la richesse de ce patrimoine lexical au service de l'expressivité mystique.

Mais le traité reflète aussi différentes formes d'énonciation de ce discours mystique et jouit ainsi d'une exceptionnelle diversité stylistique:

Le traité connaît toute la riche palette de l'expression idiomatique spirituelle: du style de traité rigide, sec à la vive exhortation, de l'instruction élémentaire à la plus haute abstraction et la plus fine spiritualité; à côté des arides cliquetis philosophiques, les tonalités d'une réchauffante et réjouissante intimité avec Dieu, plus quelques passages de pure poésie ${ }^{65}$.

Le compilator n'a donc pas cherché à retravailler et à unifier le style des diverses pièces de sa mosaïque, dont l'enchevêtrement donne au traité une configuration stylistique guère harmonieuse, contrastant quelque peu avec l'homogénéité de son agencement sys-thématique. Cette impression est singulièrement marquée par l'incorporation de séquences instaurant un dialogue entre un

61. Ibid., p. 230, 7-10; trad.: «que l'âme, en s'abîmant dans une nonrecherche, cherche le fond sans fond de la Déité».

62. Voir notamment Benno Schmoldt, Die deutsche Begriffssprache Meister Eckharts. Studien zur philosophischen Terminologie des Mittelhochdeutschen, Heidelberg, Quelle \& Meyer, 1954, p. 49-62.

63. Compilatio mystica, éd. R. Cadigan (n. 10), p. 233, 14-15; trad. : «dans la mer sans fond de la Déité».

64. Ibid., p. 416, 22-417, 1; trad.: «dans la source sans fond de la nature divine $\gg$.

65. J. Seitz, Der Traktat des 'Unbekannten dt. Mystikers' (n. 11), p. 91. 
disciple et son maître (selon le procédé question-réponse: Der junger frage/sprach [...] Der maister antwúrtet), donnant alors au traité sa coloration didactique. Tout le début de la compilation (fol. 1r-19r) consiste ainsi en un long entretien doctrinal rapporté au style direct (par exemple: Nun frage ich..., Die antwurt: Waist $d u$ nit, das...) et se ponctuant en étroite conformité avec l'incipit ${ }^{66}$, si bien qu'on remarque automatiquement qu'il s'agit là d'une seule entité textuelle indépendante (qui plus est, les fragments juxtaposés immédiatement après abandonnent ce rapport d'interlocution «je-tu»). Cette variété stylistique répond donc logiquement au caractère compilatoire du traité, c'est-à-dire à une reprise quasi textuelle d'un nombre considérable d'extraits.

66. Voir Compilatio mystica, éd. R. Cadigan (n. 10), p. 37, 2-8: «Vnd also hast du kurtzlichen gemerket, wie der mensche nach dem gotlichen bilde geschaffen ist, als got sprach: „Wir machent den menschen nach vnserm bilde vnd gelichnússe“». Voir ci-dessus, n. 41. On a en outre longtemps supposé que l'ensemble du passage, faute d'identification, était de la main même du compilator (voir Honemann, «Lehrsystem» [n. 14], col. 678). Or, il s'agit là en fait d'un long extrait du premier livre, chapitre 1, du traité «Der menschen adel, val vnd erlösung» (aussi connu sous le nom d' «Audi filia-Dialog»), attribué au bien-nommé Meister des Lehrgesprächs. Ce traité n'est recensé que dans un seul manuscrit, le manuscrit CPC 1945 de la Bibliothèque municipale de Colmar. Les fol. 1r-19r de la Compilatio mystica correspondent alors aux fol. 6rb-11va du manuscrit de Colmar. Pour une description du ms., voir Balázs J. Nemes, «Dis Buch ist iohannes schedelin. Die Handschriften eines Colmarer Bürgers aus der Mitte des 15. Jahrhunderts und ihre Verflechtungen mit dem Literaturangebot der Dominikanerobservanz», dans Barbara Fleith, Rene Wetzel (éd.), Kulturtopographie des deutschsprachigen Südwestens im späteren Mittelalter. Studien und Texte, Berlin-New York, De Gruyter, 2009 (Kulturtopographie des alemannischen Raums, 1), p. 157-214, ici p. 201. Voir également Karl Heinz Witte, Der Meister des Lehrgesprächs und sein 'In-principio-Dialog'. Untersuchung und Edition, Munich, Artemis, 1989 (Münchener Texte und Untersuchungen zur deutschen Literatur des Mittelalters, 95), p. 148-161. L'étude de Witte nous a alors permis d'identifier un autre passage dans la troisième partie de la Compilatio mystica: éd. R. Cadigan (n. 10), p. 426, 18-427, 15 = Ms. CPC 1945, fol. 99vb (voir Witte, p. 173). Il est donc à supposer que d'autres passages non identifiés de la Compilatio mystica empruntent également au traité susmentionné du Meister des Lehrgesprächs. 


\section{Une mosaïque mystique}

Les travaux successifs de Pahncke ${ }^{67}$, Spamer ${ }^{68}$, Strauch $^{69}$ et Seitz $^{70}$ ont jusqu'ici permis d'identifier deux tiers des matériaux textuels qui composent ce traité-mosaïque. Sans surprise, l'auteur le mieux représenté est de loin Maître Eckhart. Jusqu'à présent, plus de 140 parallèles ont, en effet, été établis (sur l'ensemble des deux versions de la compilation $)^{71}$, dont l'essentiel correspond à des extraits de Predigten, pour la plupart reconnus désormais comme authentiques. La Compilatio mystica peut dès lors, comme chaque traité-mosaïque, se révéler d'un précieux recours pour l'authentification de nouveaux sermons en tant que « témoins textuels de second degré $»^{72}$, de sorte qu'elle mériterait d'être intégrée à la Gesamtausgabe du maître rhénan au titre de «recueil d'extraits représentatif ${ }^{73}$. Le reste des emprunts eckhartiens provient de textes dont l'authenticité n'est pas avérée, ou bien considérés comme apocryphes: Traktate, Sprüche, Liber positionum. Bien que la présence de matériaux textuels eckhartiens soit attestée de manière substantielle dans les trois parties du traité, on constate toutefois une forte concentration dans la seconde, démontrant toute la prééminence accordée à l'enseignement d'Eckhart concernant les questions d'ordre spéculatif. C'est en outre au début de la seconde partie que l'on recense la majorité des passages empruntés à Suso, puisés intentionnellement, d'une part, dans la Vita aux chapitres 48-49 et surtout 50-52, qui constituent le point culminant de la

67. Voir Max Pahncke, Untersuchungen zu den deutschen Predigten Meister Eckharts, Diss., Halle, Karras, 1905, p. 6-11.

68. Voir A. Spamer, «Zur Überlieferung der Pfeifferschen Eckharttexte» (n. 19) et Über die Zersetzung (n. 8).

69. Voir Philipp Strauch, «Zu Greiths Compilatio mystica», dans Albert Ahn (éd.), Hundert Jahre A. Marcus und E. Webers Verlag 1818-1918, Bonn, Marcus \& Weber, 1919, p. 132-135.

70. Voir J. Seitz, Der Traktat des 'Unbekannten dt. Mystikers' (n. 11), p. 53-70 [tableau].

71. Voir le tableau (xls) récemment établi par E. Triebel sur le site internet www.eckhart. de.index.htm? wirkung.htm

72. G. Steer, «Echtheit und Authentizität der Predigten Meister Eckharts», dans G. Stötzel (éd.), Germanistik - Forschungsstand und Perspektiven. Vorträge des Deutschen Germanistentages 1984, II, Berlin-New York, De Gruyter, 1985, p. 41-50, ici p. 43.

73. Id., «Die Gesamtausgabe der deutschen und lateinischen Werke Meister Eckharts. Konzept und Durchführung», Heinrich-Seuse-Jahrbuch, 4 (2011), p. 113-166, ici p. 155. 
spéculation susienne en répondant aux questions fondamentales was ist got, ald wa ist got, ald wie ist got $?^{74}$, thématisées dans cette section du traité ${ }^{75}$; puis, d'autre part, dans le Petit livre de la Vérité aux chapitres I et III-5 (dont le contenu doctrinal renvoie aux chapitres susmentionnés dans la Vita), afin notamment d'accentuer l'arrière-plan dionysien de l'enseignement d'Eckhart sur l'inconcevabilité et l'indicibilité de la gotheit - auquel Suso fait alors écho. Quant à Tauler, le traité lui est surtout redevable dans la troisième partie, relative aux instructions spirituelles et ascétiques données à l'âme pour suivre le chemin conduisant à l'unio mystica; ce qui tend à montrer que ce prédicateur rhénan était particulièrement estimé pour sa pédagogie de directeur spirituel ${ }^{76}$. Du reste, on notera que cette mosaïque est composée de 32 extraits du Livre de la pauvreté spirituelle, un célèbre traité mystagogique anonyme, considéré comme pseudo-taulerien ${ }^{77}$. Compilant de la sorte nombre de fragments tirés d'autres traités pseudépigraphes (par exemple Vom Wesen Gottes, Von der wúrkunge der sele, Diu reissunge und die bewissunge zu dem beschouwende lebende), la Compilatio mystica livre un assez bon aperçu de la production littéraire née de l'influence exercée au XIV ${ }^{\mathrm{e}}$ siècle par Maître Eckhart et ses disciples ${ }^{78}$.

Ces multiples et évidentes attaches (thématiques, linguistiques, textuelles) du traité avec l'école eckhartienne permettent alors d'acquiescer à la remarque d'Otto Karrer selon laquelle «l'auteur est sûrement un typique défenseur de la mystique d'Eckhart». «Et le plus intéressant»-ajoute Karrer - «est que, passant complètement sous silence l'infortune du Maître, il le cite avec la plus grande estime aux côtés du lieben St. Thomas, Bischof Albrecht et dem von Sterngassen (un mystique contemporain du même cercle) en tant

74. Seuses Leben, c. 50, ed. Bihlmeyer (n. 1), p. 171, 3-5; trad. J. AnceletHustache (n. 1), p. 289: «Dites-moi ce qu'est Dieu, où est Dieu et comment est Dieu».

75. La première des trois questions est reprise textuellement à Suso; voir Compilatio mystica, éd. R. Cadigan (n. 10), p. 127, 1-7.

76. Voir Kurt Ruh, Geschichte der abendländischen Mystik, III: Die Mystik des deutschen Predigerordens und ihre Grundlegung durch die Hochscholastik, Munich, C. H. Beck, 1996, p. 491.

77. Voir ibid., p. 517-526.

78. Dans la Compilatio mystica, on signalera toutefois la reprise d'extraits de sermons d'autres prédicateurs mystiques allemands n'étant pas reconnus comme disciples d'Eckhart, par ex. Jean Franco [de Cologne] et le Kraft de Boyberg. 
que maître de vie spirituelle ${ }^{79} \gg$. Représentant de l'école dominicaine allemande le plus souvent nommé dans la Compilatio mystica (sept fois ${ }^{80}$ ), Eckhart y joue par conséquent un double rôle: d'une part, en tant que source principale de l'enseignement systématisé par le traité et des fragments compilés dans ce but, puis, d'autre part, en tant qu'autorité invoquée expressément dans le texte en qualité de «maister».

\section{L'érudition scolastique du Lebemeister}

À l'instar de la plupart des disciples d'Eckhart, l'auteur de la Compilatio mystica s'applique donc avant tout à mettre en valeur la doctrine spirituelle du «Maître ${ }^{81}$ et, afin d'en garantir l'autorité contre les incompréhensions et les attaques résultant de sa condamnation, à recopier des passages faisant ressortir de manière singulière le lien qu'entretient la mystique rhénane avec la tradition philosophique et théologique. Le discours mystique véhiculé par le traité est en effet constamment appuyé et, par suite, cautionné par le recours exprès à d'autres auctoritates (païennes et chrétiennes, grecques et latines, antiques et médiévales): Aristote (par l'intermédiaire des philosophes arabes, surtout d'Avicenne), Sénèque, Origène, Jean Chrysostome, Ambroise, Jérôme, Augustin, Proclus, le pseudo-Denys l'Aréopagite, Grégoire le Grand, Maxime le Confesseur, Jean Damascène, Anselme de Cantorbéry, Bernard de Clairvaux, Hugues et Richard de Saint-Victor, Albert le Grand

79. O. Karrer, «Aus einer mittelalterlichen Mystikerhandschrift» (n. 21), p. 7.

80. Voir Compilatio mystica, éd. R. Cadigan (n. 10), p. 94, 10; p. 162, 10-11 et $21-22 ;$ p. 180,$13 ;$ p. 300, 20-21; p. 306, 6-7; p. 424, 9. Sur les sept références explicites à maister e [c]khart, une seule n'a pu jusqu'alors être identifiée. D'autre part, la première d'entre elles est fautive (p. 94, 10), puisqu'elle introduit un passage d'un sermon allemand de Nicolas de Strasbourg (voir Predigt VI, dans Franz Pfeiffer [éd.], Deutsche Mystiker des vierzehnten Jahrhunderts, I: Hermann von Fritzlar - Nikolaus von Strassburg - David von Augsburg, Leipzig, G. J. Göschen, 1845, p. 276, 2-9). D'autres extraits de traités et sermons allemands de ce frère prêcheur - lector à Cologne et défenseur d'Eckhart lors de son procès - ont aussi été identifiés dans la Compilatio mystica; voir Eugen Hilenbrand, Nikolaus von Strassburg: Religiöse Bewegung und dominikanische Theologie im 14. Jahrhundert, Fribourg en Brisgau, Karl Alber, 1968, p. 132-133.

81. Cela permettrait d'expliquer pourquoi les autres mystiques rhénans utilisés dans le traité ne sont pas expressément nommés. 
et Thomas d'Aquin ${ }^{82}$. À travers cette accumulation d'autorités transparaît tout le poids de la formation scolaire et de l'érudition scolastique dont se nourrit l'enseignement spéculatif des mystiques rhénans, moyennant «un véritable transfert culturel qui s'opère dans la prédication allemande d'Eckhart ${ }^{83}$, lequel détache alors ces supports "autoritaires" de leur cadre d'énonciation institutionnel (ou "magistral") d'origine:

Du même coup, c'est tout le système de références conceptuelles des maîtres universitaires, leur culture, leur univers de lecture qui se trouvent traduits. C'est là ce qui fait la spécificité de la mystique rhénane: avec elle [...] l'ensemble profondément hétérogène des sources qui forment le champ d'énoncés disponibles à la pratique discursive des maîtres $[\ldots]$ sor $[\mathrm{t}]$ de l'université ${ }^{84}$.

Bousculant dans ce sens le clivage entre théologie mystique et théologie scolastique, la Compilatiomysticaillustre ainsile faitque «les mystiques [rhénans] sont justement aussi, et au sens propre du terme, les premiers scolastiques [allemands] $\gg^{85}$. À cet égard, on constatera par exemple que, sur la question scolastique du primat accordé à l'intellect ou à la volonté et l'amour (un topos de l'anthropologie), le traité reste en prise étroite et directe avec le contexte universitaire, se référant à la controverse doctrinale qui opposa sur ce point les maîtres dominicains et franciscains: une dispute d'École qualifiée de groser krieg $^{86}$ (et dont l'obrost vernunft aura immanquablement le dernier mot, en conformité avec l'orthodoxie dominicaine).

Mais bien qu'imprégnée de la culture savante, la Compilatio mystica rappelle néanmoins les limites ou l'insuffisance de l'ensei-

82. L'ensemble représente près de 170 mentions explicites. Les auteurs les plus invoqués sont le pseudo-Denys (50 fois) et Augustin (48 fois). À cela s'ajoute également près d'une quarantaine de références anonymes du type ain maister ou die maister, ou bien encore ain haidescher maister (« un maître païen»), en faisant notamment référence à Avicenne. La résurgence de ces trois grandes auctoritates (Denys, Augustin, Avicenne) dans la Compilatio mystica s'explique alors par le fait qu'elles forment en substance l' «héritage albertinien », c'est-à-dire la base philosophico-théologique sur laquelle se constitue la spiritualité mystique rhénane; voir sur ce point Alain de Libera, Introduction à la mystique rhénane: d'Albert le Grand à Maître Eckhart, Paris, O.E.I.L., 1984, p. 37-56.

83. A. de Libera, «Sermo mysticus» (n. 52), p. 49.

84. Ibid.

85. Ibid., p. 44.

86. Compilatio mystica, éd. R. Cadigan (n. 10), p. 114, 14-115, 2: «Nun ist ain frage vnder den maister, welles fúr loufe vnder verstantnússe vnd minne. [...] Vnd hier vnder so ist ain groser kriege ». 
gnement des théologiens de métier pour s'enquérir du mystère divin :

C'est pourquoi un Maître [scil. Eckhart] dit: Tous ceux de Paris et tous ceux de Bologne ou encore ceux de Padoue ne peuvent comprendre, avec toutes leurs puissances et leurs sciences, ce que Dieu est dans la moindre créature, de même que le monde entier ne peut le comprendre. Car tout ce que l'on peut penser de Dieu, ce n'est pas totalement Dieu. Ce que Dieu est en Lui-même, personne ne peut y parvenir, à moins d'être ravi dans une lumière qui est Dieu Lui-même ${ }^{87}$.

Notons alors qu'en reprenant là un fragment du Sermon allemand 100 d'Eckhart $^{88}$, le compilateur y substitue volontairement la phrase initiale, laquelle suggérait l'identité du célèbre prédicateur rhénan - moyennant une référence autobiographique faisant semblet-il allusion à l'un de ses deux magistères parisiens: «Quand je prêchais à Paris, j' ai dit ainsi - et j'ose bien le [re]dire $-\gg^{89}-$, par une périphrase anonymisante: «C'est pourquoi un maître dit». Qui plus est, il supprime l'expression caractéristique «Mais je dis maintenant» (qui, dans la version authentique du sermon, introduit la remarque suivante: «le monde entier ne peut le concevoir»), si bien que la reprise de ce passage dans la Compilatio mystica ne rend plus compte d'une révision par Eckhart lui-même de ses dires antérieurs. Selon Hasebrink - qui a constaté un modus excerptionis similaire dudit fragment dans un autre traité de compilation -, «le compilateur extrait donc dans ce cas une pièce de sa mosaïque, sans se heurter aux affirmations du contexte originaire [du sermon] $\gg^{90}$. Tout aussi remarquable est l'actualisation qu'il opère de la critique faite ici par Eckhart aux théologiens de métier, en tant qu'elle ne s'adresse désormais plus exclusivement à ceux de Paris, mais aussi à ceux de Bologne et de Padoue. Cet ajout du compilateur répercute ainsi dans le texte l'essor qu'ont connu entre-temps les universités italiennes, notamment celles de Bologne et de Padoue, qui comptèrent parmi les plus prestigieuses d'Europe à la fin du

87. Compilatio mystica, éd. R. Cadigan (n. 10), p. 151, 24-152, 8: «Wan es sprichet ain maister: Alle die von pari(n)s vnd alle die von polonie noch die von badouwe mugent nit begriffen mit allen iren kreften vnd kúnsten, was got in der minsten creature sige, noch alle diese welt mag es nit begriffen ».

88. Voir Pr. 100, DW, IV/1, Stuttgart, Kohlhammer, 2003, p. 277, 1. 51-56.

89. Ibid., p. 277, 51-52: «So ich ze Parîs predige, sô spriche ich - und ich getar es wol sprechen $-\gg$.

90. Hasebrink, «Zersetzung» (n. 2), p. 83-84. 
$\mathrm{XIV}^{\mathrm{e}}$-début du $\mathrm{XV}^{\mathrm{e}}$ siècle, période à laquelle fut composé le traitémosaïque ${ }^{91}$.

Dès lors, si la Compilatio mystica, dans son effort de systématisation de la mystique (moyen-haut) allemande, et plus spécialement rhénane (via sa figure de proue), en dénote la part qui ressortit à l'enseignement abstrait du Lesemeister et à son érudition scolastique, c'est aussi et surtout pour montrer - suivant l'intention avouée de l'eckhartisme - que cette doctrine spéculative trouve sa légitimité et sa véritable expression mystique dans une expérience intérieure vécue, à laquelle nous conduisent les instructions spirituelles du Lebemeister $^{22}$.

Qu'il suffise donc, pour conclure, de reprendre la métaphore dont se sert le titre de notre étude et de constater que le doux miel collecté dans la Compilatio mystica a un goût très prononcé de mystique rhénane, émanant du nectar de sa fleur la plus éclatante - Maître Eckhart -, s'enracinant certes dans le terreau de la théologie latine scolastique (ce qui lui donne alors un certain coloris traditionnel), mais trouvant son épanouissement et répandant ainsi son parfum pénétrant et extatique dans une prédication en moyen haut-allemand, c'est-à-dire dans un discours «déprofessionnalisé» exhortant chacun à laisser advenir le seul «Maître intérieur».

mauriegm@uni-koeln.de

91. Ces brèves remarques liées à ce fragment eckhartien nous permettent alors d'apprécier quelque peu la contribution personnelle du compilateur (outre le plan d'ensemble et la mise en ordre des textes utilisés), en nous renseignant sur le modus excerptionis (décontextualisant) et le modus compilationis (recontextualisant) ici appliqués.

92. On peut effet établir un net parallèle entre le fragment de la Compilatio mystica cité ci-dessus (n. 79) et le plus célèbre des Dits attribués à Maître Eckhart: «Ez sprichet meister Eckehart: wêger wêre ein lebemeister denne tûsent lesemeister; aber lesen unde leben ê got, dem mac nieman zuo komen. Solte ich einen meister suochen von der geschrift, den suohte ich ze Parîs und in hôhen schuolen umbe hôhe kunst. Aber wollte ich frâgen von vollekomenem lebenne, daz kunde er mit niht gesagen» (Sprüche, Nr. 8, dans Franz Pfeiffer [éd.], Deutsche Mystiker des vierzehnten Jahrhunderts, II: Meister Eckhart, Leipzig, G. J. Göschen, 1857, p. 599, 19-24). 